
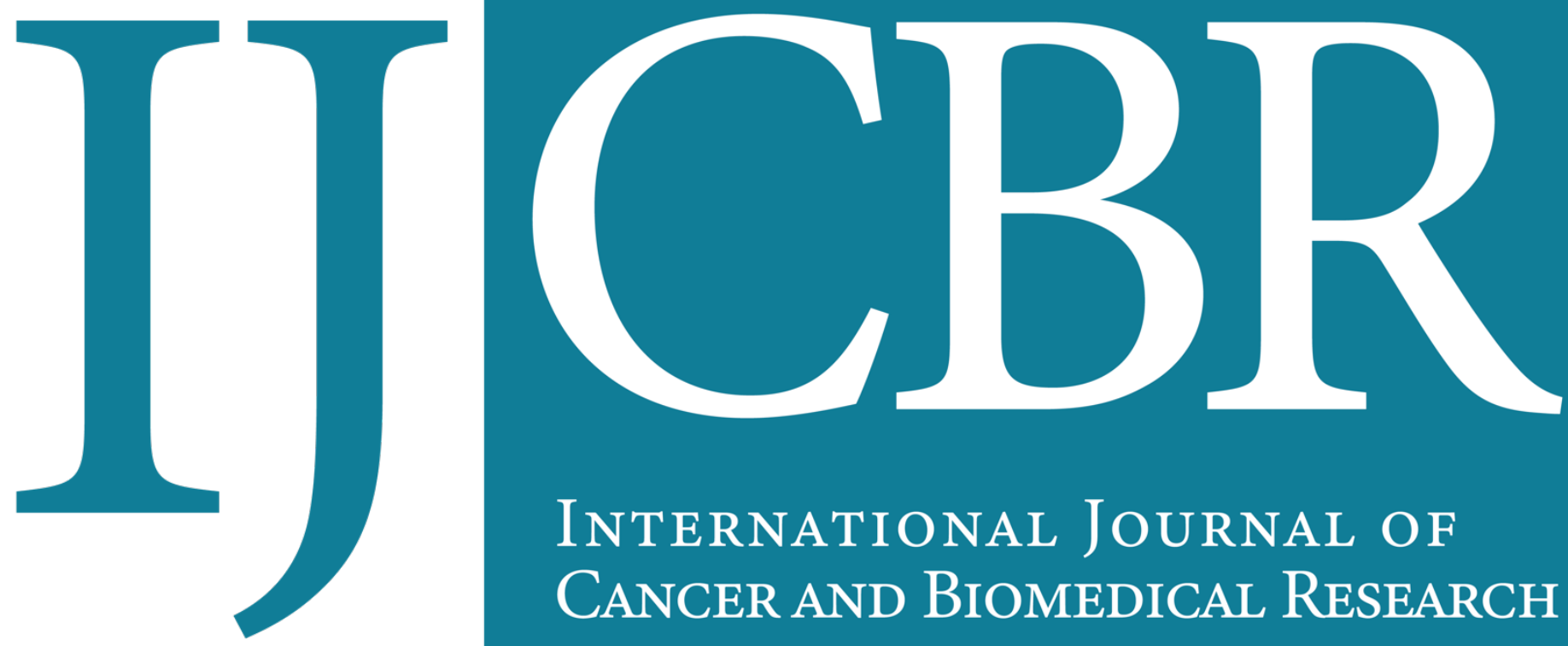

INTERNATIONAL JOURNAL OF CANCER AND BIOMEDICAL RESEARCH

https://jcbr.journals.ekb.eg

Editor-in-chief

Prof. Mohamed Labib Salem, PhD

Optimum initiation-time of direct-acting antivirals for hepatitis $\mathbf{C}$ after curative ablation of hepatocellular carcinoma improves neoplastic recurrence-free survival

Ahmed A. Elhadidy, Ahmed F. Selim, Mohamed E. Sarhan, Mohamed M. Mabrouk, Heba M. Samier and Abdallah A. Elsawy 


\title{
Optimum initiation-time of direct-acting antivirals for hepatitis C after curative ablation of hepatocellular carcinoma improves neoplastic recurrence-free survival
}

\author{
Ahmed A. Elhadidy ${ }^{1}$, Ahmed F. Selim ${ }^{1}$, Mohamed E. Sarhan ${ }^{1}$, Mohamed M. Mabrouk ${ }^{1}$, Heba M. Samier ${ }^{2}$ and \\ Abdallah A. Elsawy ${ }^{1}$ \\ ${ }^{1}$ Department of Internal Medicine, Faculty of Medicine, Tanta University, Tanta, Egypt \\ 2Department of Diagnostic Radiology, Faculty of Medicine, Tanta University, Tanta, Egypt
}

\section{III}

Background: Early initiation of direct-acting antivirals (DAAs) after ablation of hepatocellular carcinoma (HCC) may boost the proliferation of the potentially present extra-lesion dormant neoplastic micro-nodules. Aim: We evaluated the performance of early initiation of DAAs therapy after curative ablation of HCC as a risk factor for early neoplastic recurrence in chronic hepatitis-C patients, aiming to select an optimum initiation time for DAAs therapy. Materials and Methods: This retrospective cohort study was conducted at hepatology unit at Tanta university Hospitals in the period from January 2016 to January 2020 on 142 naïve HCV patients who achieved SVR to DAAs therapy after complete ablation response of HCC. Postablation to DAAs-initiation elapsed time window were estimated. All patients were followed up regularly for 18 months from completion of DAAs therapy for the ascertainment of HCC recurrence. Results: $\mathrm{HCC}$ recurrence occurred in $27.5 \%$ of our patients, most of them (43.6\%) in the first 6 months post-DAAs therapy with an incidence rate of 1 per100 persons-months. Post-ablation to DAAs-initiation time window had a significant performance as a risk factor for early HCC recurrence after DAAs therapy (AUROC:0.8887; $\mathrm{Cl}: 0.821-0.953$; sig:0.000), the tenth month of postablation time was the most accurate cutoff value for ruling out $\mathrm{HCC}$ recurrence for values more than it (sensitivity: 97.4\%, LR-:0.09). Conclusions: Early DAAs-initiation after curative HCC ablation seems to be a significant risk factor for early HCC recurrence; DAAs-initiation should be delayed after 10 months.

Keywords: Direct Acting Antivirals, Hepatocellular Carcinoma, Initiation Time, Risk Factor, Recurrence
III ARTICLE INFO

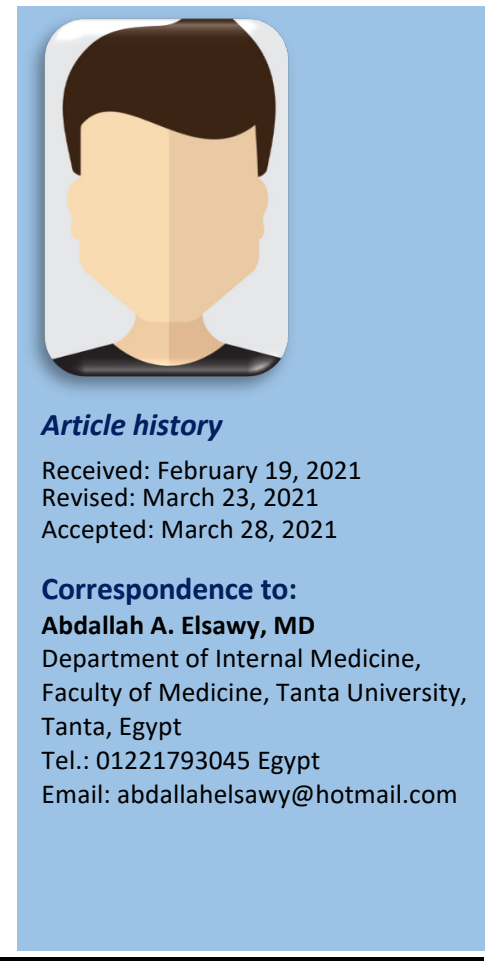

Editor-in-Chief: Prof. M.L. Salem, Ph.D. - Article DOI: 10.21608/jcbr.2021.64062.1182

\section{INTRODUCTION}

Successful DAAs therapy for chronic hepatitis (CHC) has a beneficial effect in the prevention of cirrhosis progression and decompensation; accordingly, it may reduce the risk of HCC incidence, however, an extensive debate is still ongoing around the possible increase of HCC recurrence after curative ablation in $\mathrm{CHC}$ patients who received DAAs therapy even after the sustained virological response (SVR) (European Association for the Study of the Liver, 2018; El Kasssas et al., 2019; Saraiya et al., 2018; Reig et al., 2016). This debate encouraged many researchers to study the possible risk factors that may be linked to the increased HCC recurrence after DAAs therapy hoping to explain and introduce suitable solutions to mitigate this phenomenon, most of these risk factors were related to the degree of liver fibrosis, reserve liver function, tumor burden, alpha-fetoprotein (AFP) as well as the interval time from ablation to DAAs initiation (Colombo et al., 2018; Guarino et al., 2018; Reig et al., 2017; Baumert et al., 2017; Piscaglia, et al., 2017; Tampaki et al., 2018).

Early recurrence of HCC after DAAs therapy for $\mathrm{CHC}$ may be attributed to multiple explanations, mostly related to incomplete ablation or the potential presence of intrahepatic extra-lesion micro-metastasis (Sherman, 2008). DAAs 
induced immune reconstitution by abrupt virus clearance seems to open a transitory immunosuppressive phase (Meissner et al., 2014; Serti et al., 2015; Chu et al., 2017; vanWilgenburg et al., 2016); accordingly, these dormant tumor micro-nodules or micrometastasis might find an ideal condition to proliferate and become radiologically apparent. The equilibrium between both cancer cell proliferation and lysis may be achieved by changes in the immune system and/or angiogenesis and it seems to be the leading cause of this tumor mass dormancy (Teng et al., 2008; Sanduzzi-Zamparelli et al., 2019).

Based on these explanations, we hypothesized that early initiation of DAAs after ablation may boost the proliferation of these dormant neoplastic micro-nodules, so, the post-ablation to DAAs initiation elapsed time may share an important link to the possibility of HCC recurrence after starting of DAAs therapy. We focused in our study on this elapsed time parameter to evaluate its performance as a risk factor for early neoplastic recurrence after DAAs therapy for HCV aiming to select an optimum time to start DAAs therapy after curative ablation of HCC.

\section{MATERIALS AND METHODS}

\section{Ethical consideration}

We conducted this study in accordance with the declaration of Helsinki and our institutional ethics committee.

\section{Study design}

This was a retrospective cohort study that was conducted in accordance with the Transparent Reporting of a multivariable prediction model for Individual Prognosis Or Diagnosis (TRIPOD) Statement (Collin et al., 2015).

\section{Study setting and participants}

This study was conducted at the hepatology unit at Tanta University Hospital (a tertiary hospital). We reviewed the registered data for all $\mathrm{CHC}$ patients (who were already received DAAs therapy after ablation of HCC with a complete response and who were followed up periodically at our outpatient clinic in the period from January 2016 to January 2020) for potential eligibility criteria $(n=189)$.
In our study, we focused on the potential link between the post-ablation to DAAs-initiation elapsed time window and HCC recurrence, so, we tried to enhance our exclusion criteria to exclude other known risk factors for HCC recurrence; 7 patients were excluded due to the combined $\mathrm{HCV} / \mathrm{HBV}$ affection, 11 patients were excluded as they had previous HCV treatment experience, 4 patients were excluded due to failure of achievement of SVR to DAAs therapy, 4 patients were excluded as the HCC ablation modality were transarterial chemoembolisation (TACE), 6 patients were excluded due to unconfirmed complete ablation response immediately before DAAs- initiation, 2 patients were excluded as their pre-ablation criteria were not registered, 4 patients were excluded due to previous history of HCC recurrence, 12 patients were excluded due to irregular adherence to post-ablation follow up protocol and 3 patients were excluded due to the presence of non-characterized hepatic nodules immediately before DAAs-initiation. Therefore, the eligibility criteria were fulfilled in only 142 naïve HCV patients who achieved SVR to DAAs therapy after curative HCC ablation (surgical resection, radiofrequency ablation, microwave ablation or percutaneous ethanol injection) as illustrated in our study flowchart (Figure 1).

\section{Data collection}

The registered pre-ablation, post-ablation and post-DAAs follow-up data for each enrolled patient were thoroughly evaluated. The following participants' criteria were evaluated: demographic criteria (age and sex), pre-ablation laboratory criteria (alanine aminotransferase [ALT], aspartate aminotransferase [AST], s.albumin, s.bilirubin, prothrombin concentration [PC], s.creatinine, platelet count, hemoglobin, fibrosis-4 [FIB-4] score and ChildPugh score), pre-ablation HCC criteria (volume, number and alpha-fetoprotein [AFP] level), post-ablation to DAAs-initiation elapsed time window.

The assessment of HCC ablation was done using either triphasic computed tomography (TCT) or dynamic magnetic resonance imaging (DMRI) and the complete ablation response was achieved when there were no radiological criteria for remained active neoplastic areas 
within the previously ablated HCC (arterial hyper-enhancement with delayed washout) (European Association for the Study of the Liver, 2018), the last radiological assessment was done just before DAAs-initiation to rule out any HCC recurrence before the start of DAAs therapy. All our naïve $\mathrm{HCV}$ patients were treated in accordance with EASL recommendations on treatment of hepatitis $C$ using either the fixed-dose combination of sofosbuvir (400 mg) and ledipasvir (90 mg) once daily for 12 weeks or the combination of daily sofosbuvir (400 mg) and daily daclatasvir (60 $\mathrm{mg}$ ) for 12 weeks, the SVR was defined as undetectable HCV RNA in blood by a sensitive assay 12 weeks (SVR 12) or 24 weeks (SVR 24) after the treatment completion (European Association for the Study of the Liver, 2017).

\section{Follow up and study endpoint}

The registered post-DAAs follow up data were traced periodically every 3-6 months for 18 months after completion of DAAs therapy for the ascertainment of the recurrent HCC which was confirmed by using either TCT or DMRI and was defined by the reappearance of radiological criteria of active neoplastic lesions within or nearby the previously ablated mass (arterial hyper-enhancement with venous or delayed washout). The time of HCC recurrence was calculated from the registered date of ablation.

\section{Statistical analysis}

IBM SPSS; version 23 statistic software (IBM, NY, USA) was used for data summarization and statistical analysis. The mean (with standard deviation) was used for normally distributed quantitative data while the median (with minimum and maximum) was calculated for abnormally distributed quantitative data; qualitative data were cross-tabulated as frequency and relative frequency.

Cox-regression analysis was performed for the possible risk predictors for post-DAAs HCC recurrence. Candidate risk predictors in this analysis were post-ablation to DAAs-initiation elapsed time window, the pre-ablation reserve liver function using Child-Pugh score, preablation HCC criteria (volume, number and AFP) and the pre-ablation degree of liver fibrosis using fibrosis-4 (FIB-4) score. Univariate Cox- regression analysis was done first for each predictor, and then the best predictors were evaluated using the multivariate Cox-regression analysis by entering all predictors simultaneously with a stepwise backward strategy.

Receiver operating characteristic (ROC) curve was calculated for the post-ablation to DAAsinitiation elapsed time window and its area under the ROC curve (with their 95\% confidence interval $[\mathrm{Cl}]$ ) was computed, two cutoff values were selected according to the clinical rule of the predictor, that is, to rule in or rule out the risk prediction of HCC recurrence. Sensitivity, specificity, positive likelihood ratio ( $(\mathrm{LR}+)$, and negative likelihood ratio (LR-) were calculated for each cutoff point.

The post-ablation to DAAs-initiation elapsed time window was graded using the ruling out cutoff value to two risk grades; low risk, and high-risk grades, the recurrence-free survival function was illustrated using Kaplan Meier method and analyzed using Log Rank test. Pvalues less than 0.05 were considered statistically significant.

\section{RESULTS}

The main evaluated participants' criteria are illustrated in table 1 including the demographic criteria (age and sex), pre-ablation reserve liver functions using Child-Pugh score, pre-ablation values of liver fibrosis using FIB-4 score, preablation HCC criteria (tumor volume, number, AFP and treatment modality) and post-ablation to DAAs-initiation elapsed time window. The incidence rate of $\mathrm{HCC}$ recurrence was found to be 1 per 100 person-months with a total of 39 patients (27.5\%). We missed outcome data for only $8(5.6 \%)$ patients without an identified cause.

Table2 illustrated the Cox-regression analysis for our evaluated risk factors of post-DAAs HCC recurrence. Firstly the univariate Cox-regression analysis was done, the candidate risk factors in this analysis were patients' demographic criteria (age and Sex), reserve liver status; we used Child-Pugh score as a surrogate parameter for liver functions and FIB-4 score was used as a non-invasive score for liver fibrosis, criteria of HCC burden (tumor volume, number and AFP) 
as well as post-ablation to DAAs-initiation elapsed time window (sig: $0.073,0.967,0.000$, $0.000,0.000,0.536,0.001$ and 0.000 respectively). After that, multivariate Coxregression analysis was done to identify the most independent significant risk factors of $\mathrm{HCC}$ recurrence and their respective hazard ratios (HR), these predictors were the Child-Pugh score (sig: 0.002, adjusted HR: 2.184 with $\mathrm{Cl}$ : 1.346-3.542), HCC volume (sig:0.016, adjusted HR: 1.056 and $\mathrm{Cl}: 1.010-1.103)$ and postablation to DAAs-initiation elapsed time window (sig: 0.000 , adjusted HR: 0.506 and $\mathrm{Cl}$ : 0.414-0.618).

Table3 of illustrated the Receiver operating characteristics (ROC) of the post-ablation to DAAs-initiation elapsed time window and its area under the ROC curve was computed (AUC: 0.887 with $\mathrm{Cl}$ : 0.821-0.953) as illustrated in figure 2, two cutoff values were selected according to the clinical rule of the predictor; the $1^{\text {st }}$ cutoff value was $\leq 4$ months to rule in the risk prediction of HCC recurrence and it was selected in accordance to its highest specificity $(98.1 \%)$ and the highest $L R+(17.17)$, the $2^{\text {nd }}$ cutoff value was $\geq 10$ months to rule out the risk prediction of HCC recurrence and it was selected in accordance to its highest sensitivity (97.4\%) and the lowest LR- (0.09), this time window was graded using the ruling out cutoff value (10 months) to 2 grades; low risk initiation-time for periods $\geq 10$ months, high risk initiation-time for periods $<10$ months, the recurrence free survival function between those different risk grades were illustrated using Kaplan Meier method and analyzed using Log Rank test showing pairewise significant difference (sig: 0.000 with an estimated mean of recurrence free survival: 36.4 months and 24.1 months for low risk and high risk initiationtime respectively) as illustrated in figure 3. As illustrated in table 4, the incidence rate of HCC recurrence was 1.8 per100 persons-months if DAAs initiated within the high-risk time window (<10 months) and $0.17 / 100$ person-months if DAAs initiated within low-risk time window. At the same time, we found that $17 \mathrm{HCC}$ recurrent cases $(43.6 \%)$ occurred within $1^{\text {st }} 6$ months after DAAs completion, 12 (30.8\%) cases occurred within the $2^{\text {nd }} 6$ months and $10(25.6 \%)$ cases occurred within the $3^{\text {rd }} 6$ months.

\section{DISCUSSION}

We focused here in our study on the postablation to DAAs-initiation elapsed time window to evaluate its performance as a risk factor for early HCC recurrence after DAAs therapy for HCV hoping to appropriate selection of optimum starting time for DAAs therapy after curative ablation of HCC.

We found that the incidence rate of $\mathrm{HCC}$ recurrence was $1 / 100$ persons-months, however, it ranges from 0.73 to $1.7 / 100$ persons-months in other studies, (Virlogeux, et al. 2017; ANRS collaborative study group on hepatocellular carcinoma, 2016) at the same time, we found that total cases of HCC recurrence were $39(27.5 \%)$ of patients within a median post-DAAs follow up time of 18 months most of them within the $1^{\text {st }} 6$ months. Saraiya et al. (2018) found a pooled HCC recurrence rate was $21.9 \%$ (95\% Cl: $16.2 \%-28.3 \%$ ). El Kassas et al. (2018) found that HCC recurrence occurred in $37.7 \%$ of patients after a median post-DAAs follow-up period of 16 months. The variability between the results of these studies seems to be due to differences in study designs, differences in HCC ablation modalities, differences in follow up periods, geographic differences, and the wide variability in the timing of DAAs-initiation, however, the proximity of $\mathrm{HCC}$ recurrence in most patients of our study to the timing of DAAs completion could be attributed as an alarm that needed an explanation.

Early recurrence of HCC after DAAs therapy for $\mathrm{CHC}$ may be attributed to multiple explanations, mostly related to, incomplete ablation or the potential presence of extra-lesional micrometastasis (Sherman, 2008). DAAs induced immune reconstitution by abrupt virus clearance seems to open a transitory immunosuppressive phase (Chu et al., 2017; vanWilgenburg et al., 2016; Waring et al., 2016; Villani et al., 2016). Accordingly, these dormant tumor micro-nodules or micro-metastasis might find an ideal condition to proliferate and become radiologically apparent. The equilibrium between both cancer cells proliferation and lysis may be achieved by changes in the immune system and/or angiogenesis and it seems to be the leading 
Potential eligible population: $(n=189)$

HCV patients who received DAA therapy after presumed successful HCC ablation

Exclusion criteria: $(n=47)$

- Combined HCV/HBV patients ( $n=7)$

- Treatment experienced HCV patients (n=11)

- Unachievemen of SVR to DAAs ( $n=4)$

- TACE treatment modality of HCC $(n=4)$

- Unconfirmed complete ablation response immediately before DAAs initiation $(n=6)$

- Preablation patients' criteria were not registered $(n=2)$

- Previous history of HCC recurrence $(n=4)$

- Unadherence to post-ablation follow up protocol $(n=12)$

- Presence of non-characterized hepatic focal lesions $(n=3)$

Eligible population: $(n=142)$

Naive HCV patients who achieved an SVR to DAA therapy after curative HCC ablation

Assessmentof possible risk predictors of post-DAAs HCC recurrence:

- The time window between curative HCC ablation and DAAs initiation.

-Preablation tumor criteria ( volume, focality, AFP).

- Preablation Child-Pugh score and FIB4 score

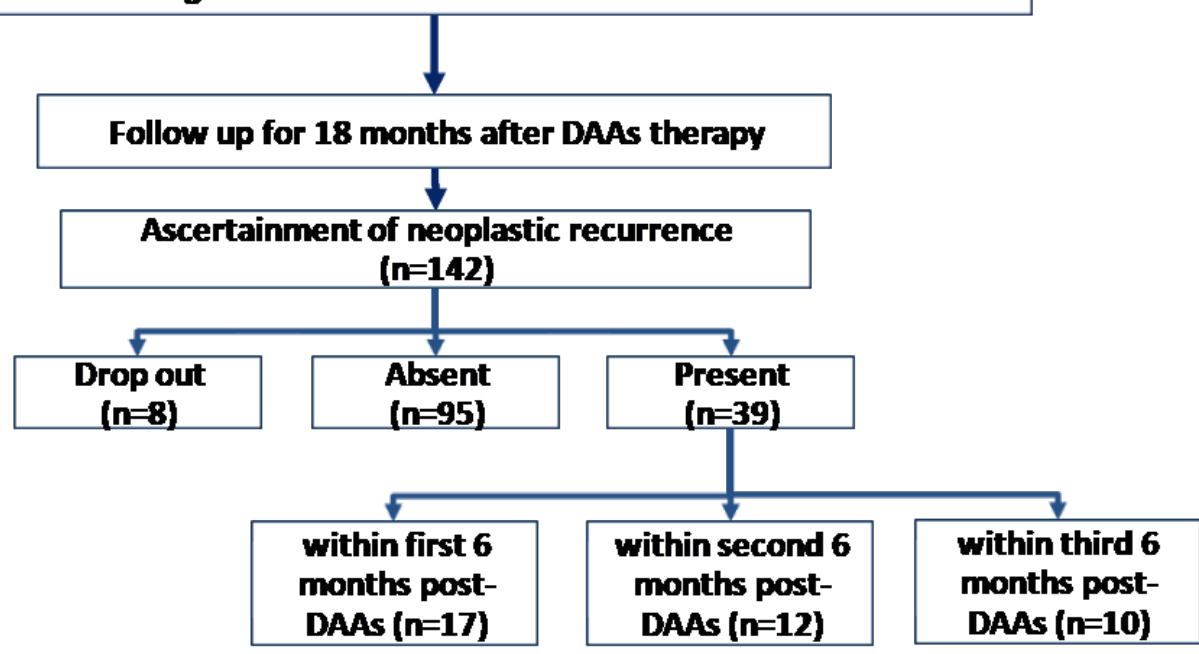

Figure 1. Study participants' flowchart. 
Table 1. Main participants' criteria

\begin{tabular}{|c|c|c|c|c|}
\hline \multicolumn{2}{|l|}{ Participants' criteria } & \multicolumn{3}{|c|}{ Value ( $n=142)$} \\
\hline \multicolumn{5}{|l|}{ Demographic criteria } \\
\hline \multicolumn{2}{|l|}{ Age (years) } & Mean (SD) & 56.12 & (7.917) \\
\hline \multirow[t]{2}{*}{ Sex } & Female & Count (\%) & 47 & $(33.1 \%)$ \\
\hline & Male & Count (\%) & 95 & $(66.9 \%)$ \\
\hline \multicolumn{5}{|c|}{ Pre-ablation laboratory parameters } \\
\hline \multicolumn{2}{|l|}{ ALT (IU/L) } & Mean (SD) & 52.26 & $(10.041)$ \\
\hline \multicolumn{2}{|l|}{ AST (IU/L) } & Mean (SD) & 47.30 & (7.656) \\
\hline \multicolumn{2}{|l|}{ S. albumin (gm/dl) } & Mean (SD) & 3.23 & $(0.280)$ \\
\hline \multicolumn{2}{|l|}{ S. bilirubin (mg/dl) } & Mean (SD) & 1.43 & $(0.256)$ \\
\hline \multicolumn{2}{|c|}{ Prothrombin concentration (\%) } & Mean (SD) & 69.72 & (6.306) \\
\hline \multirow[t]{4}{*}{ Child-Pugh score } & A5 & Count (\%) & 17 & $(12 \%)$ \\
\hline & A6 & Count (\%) & 52 & $(36.6 \%)$ \\
\hline & B7 & Count (\%) & 55 & (38.7\%) \\
\hline & B8 & Count (\%) & 18 & $(12.7 \%)$ \\
\hline \multicolumn{2}{|l|}{ FIB-4 score } & Mean (SD) & 2.49 & $(0.560)$ \\
\hline \multicolumn{2}{|l|}{ Platelets $\left(\times 10^{3} / \mathrm{mm}^{3}\right)$} & Mean (SD) & 150.42 & (18.769) \\
\hline \multicolumn{2}{|l|}{ Hemoglobin $(\mathrm{g} / \mathrm{dl})$} & Mean (SD) & 11.65 & $(0.661)$ \\
\hline \multicolumn{2}{|l|}{ S. creatinine (mg/dl) } & Mean (SD) & 1.07 & $(0.110)$ \\
\hline \multicolumn{5}{|c|}{ Pre-ablation HCC criteria } \\
\hline \multirow[t]{2}{*}{ Number } & Unifocal & Count (\%) & 76 & $(53.5 \%)$ \\
\hline & Multifocal & Count (\%) & 66 & (46.5) \\
\hline \multicolumn{2}{|l|}{ Volume $\left(\mathrm{CC}^{3}\right)$} & Median (min-max) & 27 & $(12-53)$ \\
\hline \multicolumn{2}{|l|}{ AFP } & Median (min-max) & 41.5 & $(9-1471)$ \\
\hline \multirow[t]{4}{*}{ Treatment modality } & PEI & Count (\%) & 12 & $(8.5 \%)$ \\
\hline & RFA & Count (\%) & 52 & (36.6\%) \\
\hline & MWA & Count (\%) & 70 & $(49.3)$ \\
\hline & Surgical resection & Count (\%) & 8 & $(5.6 \%)$ \\
\hline \multicolumn{5}{|c|}{ Post-ablation related criteria } \\
\hline \multicolumn{2}{|c|}{ Post-ablation to DAAs-initiation elapsed time window (months) } & Median (min-max) & 9 & $(3-16)$ \\
\hline \multirow[t]{2}{*}{ HCC recurrence } & Incidence rate & /100 persons-months & 1 & \\
\hline & Total cases & Count (\%) & 39 & $(27.5 \%)$ \\
\hline \multicolumn{5}{|c|}{$\begin{array}{l}\text { ALT: alanine aminotransferase, AST: aspartate aminotransferase, FIB-4 score: fibrosis- } 4 \text { score, HCC: hepatocellular } \\
\text { carcinoma, AFP: alpha-fetoprotein, DAAs: direct antiviral agents, PEI: percutaneous ethanol injection, RFA: } \\
\text { radiofrequency ablation, MWA: microwave ablation }\end{array}$} \\
\hline
\end{tabular}

Table 2. Cox-regression analysis for all studied risk factors of HCC recurrence

\begin{tabular}{|c|c|c|c|c|c|c|c|c|c|c|}
\hline \multirow[t]{3}{*}{ Participants' criteria } & \multicolumn{4}{|c|}{ Univariate Cox-regression analysis } & \multicolumn{6}{|c|}{ Multivariate Cox-regression analysis } \\
\hline & \multirow[t]{2}{*}{ B } & \multirow[t]{2}{*}{ SE } & \multirow[t]{2}{*}{ Wald } & \multirow[t]{2}{*}{ Sig. } & \multirow[t]{2}{*}{ B } & \multirow[t]{2}{*}{ SE } & \multirow[t]{2}{*}{ Wald } & \multirow[t]{2}{*}{ Sig. } & \multicolumn{2}{|c|}{ Adjusted HR } \\
\hline & & & & & & & & & value & $95 \% \mathrm{Cl}$ \\
\hline \multicolumn{11}{|l|}{ Demographic criteria } \\
\hline Age & 0.038 & 0.021 & 3.213 & 0.073 & & & & & & \\
\hline Male gender & 0.014 & 0.340 & 0.002 & 0.967 & & & & & & \\
\hline \multicolumn{11}{|l|}{ Reserve liver status } \\
\hline Child-Pugh score & 1.094 & 0.213 & 26.504 & 0.000 & 0.781 & 0.247 & 10.017 & 0.002 & 2.184 & $\begin{array}{l}1.346- \\
3.542\end{array}$ \\
\hline FIB-4 score & 1.197 & 0.264 & 20.505 & 0.000 & & & & & & \\
\hline \multicolumn{11}{|l|}{ HCC burden } \\
\hline HCC volume $\left(\mathrm{CC}^{3}\right)$ & 0.12 & 0.021 & 32.307 & 0.000 & 0.054 & 0.023 & 5.793 & 0.016 & 1.056 & $\begin{array}{l}1.010- \\
1.103\end{array}$ \\
\hline HCC focality & 0.198 & 0.320 & 0.382 & 0.536 & & & & & & \\
\hline AFP (ng/ml) & 0.002 & 0.000 & 12.088 & 0.001 & & & & & & \\
\hline \multicolumn{11}{|c|}{ Post-ablation to DAAs-initiation elapsed time window } \\
\hline $\begin{array}{l}\text { Post-ablation to DAAs-initiation } \\
\text { time window (months) }\end{array}$ & $\begin{array}{l}- \\
0.721\end{array}$ & 0.091 & 63.056 & 0.000 & - & 0.102 & 44.610 & 0.000 & 0.506 & $\begin{array}{l}0.414- \\
0.618\end{array}$ \\
\hline
\end{tabular}


Table 3. Receiver operating characteristics of the post-ablation to DAAs-initiation time window as a risk predictor of recurrence of hepatocellular carcinoma

\begin{tabular}{|c|c|c|c|c|c|c|c|c|c|}
\hline & \multirow{2}{*}{$\begin{array}{c}\text { Clinical } \\
\text { Rule }\end{array}$} & \multirow[t]{2}{*}{ Cutoff } & \multirow[t]{2}{*}{ Sensitivity } & \multirow[t]{2}{*}{ Specificity } & \multirow[t]{2}{*}{ LR+ } & \multirow[t]{2}{*}{ LR- } & \multicolumn{3}{|c|}{ AUROC } \\
\hline & & & & & & & Value & $(95 \% \mathrm{Cl})$ & Sig. \\
\hline \multirow{2}{*}{$\begin{array}{l}\text { Post-ablation to DAAs- } \\
\text { initiation time window } \\
\text { (months) }\end{array}$} & Rule out & $\geq 10$ & $97.4 \%$ & $27 \%$ & 1.34 & 0.09 & \multirow[t]{2}{*}{0.887} & \multirow{2}{*}{$\begin{array}{c}0.821- \\
0.953\end{array}$} & \multirow[t]{2}{*}{0.000} \\
\hline & Rule in & $\leq 4$ & $33.3 \%$ & $98.1 \%$ & 17.17 & 0.68 & & & \\
\hline
\end{tabular}

Table 4. HCC recurrence in relation to study follow up time factors

\begin{tabular}{|l|l|l|l|}
\hline Time factor & \multicolumn{2}{c|}{ Value (n=39) } \\
\hline $\begin{array}{l}\text { Post-ablation to DAAs- } \\
\text { initiation elapsed time }\end{array}$ & High-risk initiation-time (<10 months) & Incidence rate & $1.8 / 100$ persons-months \\
\cline { 2 - 4 } & Low-risk initiation-time ( $\geq 10$ months) & Incidence rate & $0.17 / 100$ persons-months \\
\hline $\begin{array}{l}\text { Post-DAAs follow-up } \\
\text { time }\end{array}$ & Within 1 ${ }^{\text {st }}$ 6 months after DAAs & Count (\%) & $17(43.6 \%)$ \\
\hline & Within 2 ${ }^{\text {nd }}$ 6 months after DAAs & Count (\%) & $12(30.8 \%)$ \\
\hline \multirow{2}{*}{ HCC: hepatocellular carcinoma, DAAs: direct antiviral agents } & Count (\%) & $10(25.6 \%)$ \\
\hline
\end{tabular}

cause of this tumor mass dormancy (Teng et al., 2008; Sanduzzi-Zamparelli et al., 2019). So, we could hypothesize that early initiation of DAAs after ablation may boost the proliferation of the potential dormant extra-lesional neoplastic micro-nodules so, the post-ablation to DAAs initiation elapsed time may share an important link to the possibility of HCC recurrence after starting of DAAs therapy.

The Cox-regression analysis of our results identified that the most independent significant risk factors for early HCC recurrence were the shorter post-ablation to DAAs-initiation time window, larger HCC volume, and higher ChildPugh score. Multiple risk predictors for HCC recurrence after DAAs therapy were evaluated in many studies with variable results, most of these risk factors were related to the degree of liver fibrosis, reserve liver function, tumor burden (size and number), AFP as well as the elapsed time from ablation to start of DAAs therapy (Colombo et al., 2018; Guarino et al., 2018; Reig et al., 2017; Baumert et al., 2017; Piscaglia et al., 2017; Tampaki et al.,(2018).

We found that, the post-ablation to DAAsinitiation elapsed time window had an accurate and significant performance in risk prediction of $\mathrm{HCC}$ recurrence after DAAs therapy as described by its Receiver operating characteristics (AUC: 0.887; $\mathrm{Cl}: 0.821-0.953$; sig: 0.000), at the same time we tried to select a suitable starting time point for DAAs therapy through selecting two cutoff values of this elapsed time window; the $1^{\text {st }}$ cutoff value was found to be $\leq 4$ months to rule in the risk prediction of $\mathrm{HCC}$ recurrence and it was selected in accordance to its highest specificity (98.1\%) and the highest LR+ (17.17), the $2^{\text {nd }}$ cutoff value was found to be $\geq 10$ months to rule out the risk prediction of HCC recurrence and it was selected in accordance to its highest sensitivity $(97.4 \%)$ and the lowest LR(0.09), this time window was graded using the ruling out cutoff value to 2 grades; low risk time window for periods $\geq 10$ months and high risk time window for periods $<10$ months with a significant difference as regard the recurrence free survival distribution (sig: 0.000), the incidence rate of HCC recurrence was found to be $1.8 / 100$ persons-months if DAAs-initiation was within the high risk time window while it was $0.17 / 100$ persons-months if DAAs-initiation was within the low risk time window. These results were confirmed by that identified by many papers that found that shorter elapsed periods from ablation to DAAs-initiation were more significant than longer time post-ablation periods however with variable cutoff time points (Ogawa et al., 2018; Minami et al., 2016; Kolly et al., 2017; Bielen et al., 2017). However, El kassas et al., (2018) found that there was no significant difference in the rate of HCC recurrence between HCV patients who received DAAs within the first 3 months post-ablation time window and those who received it later in the time window (more than 6 months). 


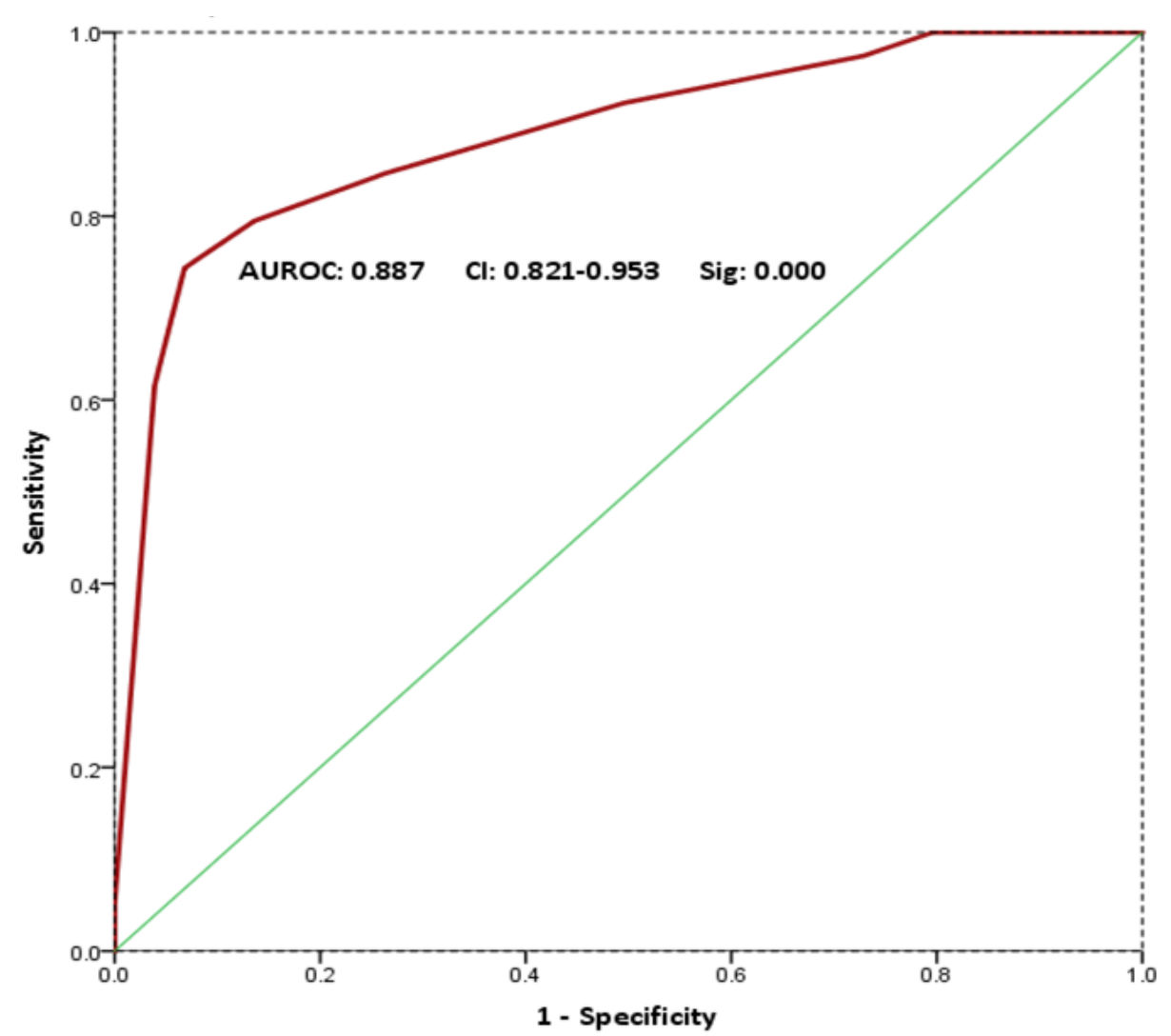

Figure 2. ROC curve for the post-ablation to DAAs-initiation time window in relation to HCC recurrence

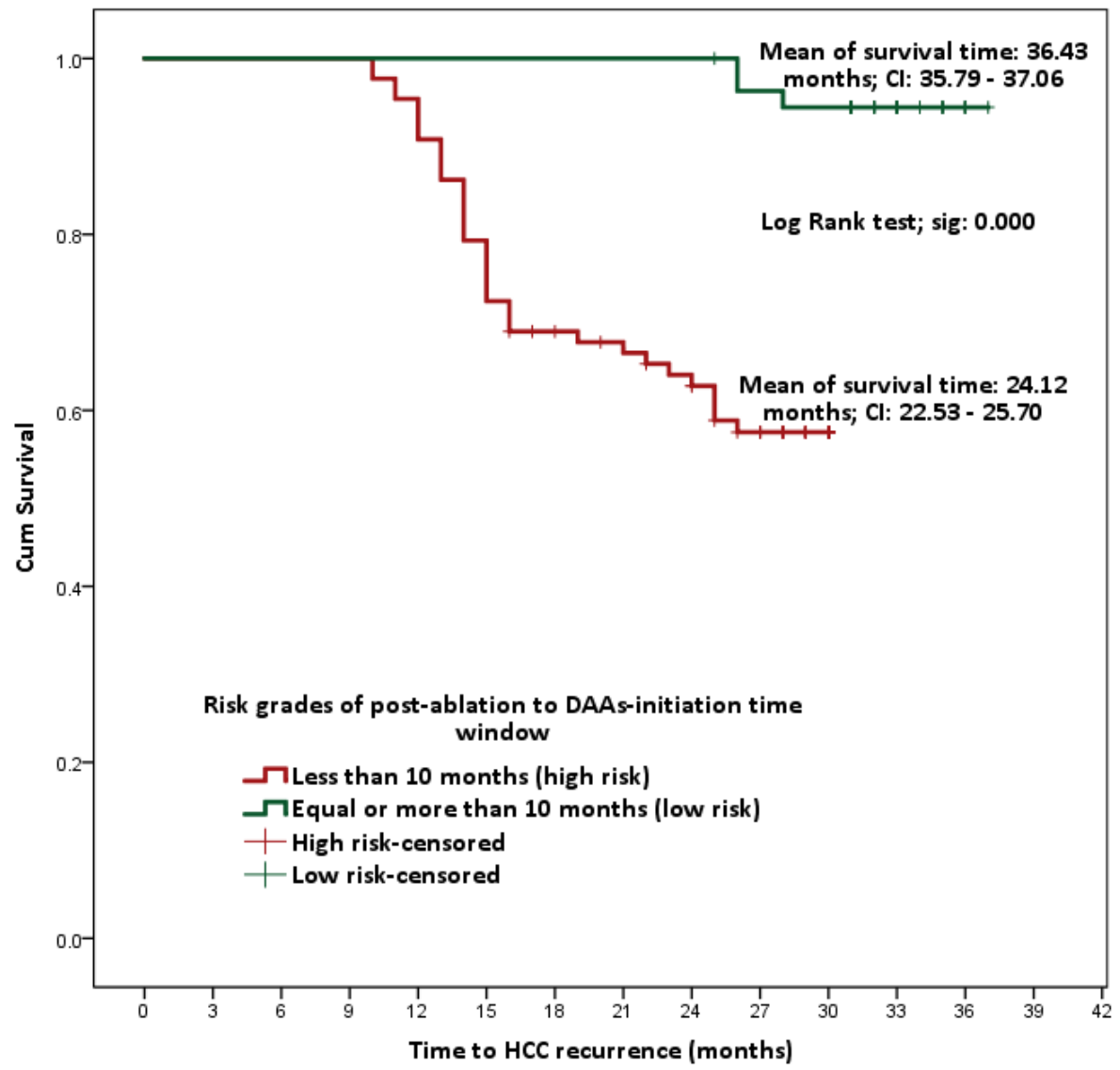

Figure 3. The Kaplan Meier of HCC recurrence-free survival function for the different risk grades of DAAs initiation-time 
Based on these explanations, we could hypothesize that early initiation of DAAs after HCC ablation may boost the proliferation of potentially present extra-lesional dormant neoplastic micro-nodules so, the post-ablation to DAAs initiation elapsed time may share an important link to the possibility of HCC recurrence after starting of DAAs therapy

Our study has some limitations; the retrospective data collection is one of these limitations, however, we tried to enhance our participants' selection and exclusion criteria to focus on our research question. This study is a uni-centric study, which affects the external generalizability of our results, and at the same time, we couldn't identify some risk factors in most of the participants' criteria as diabetes mellitus related data and body mass index. Finally, we did not include the different patterns of HCC recurrences hoping to be included in other wider studies.

We could conclude that Early DAAs-initiation after curative HCC ablation seems to be an important significant risk factor for early HCC recurrence after DAAs therapy; DAAs initiation should be delayed after 10 months from curative HCC ablation to rule out the presence of post-ablation dormant neoplastic nodules and the potential post-DAAs HCC recurrence.

\section{ACKNOWLEDGEMENTS}

The authors thank staff members in the Hepatology Unit of the Internal Medicine Department at Tanta University Hospital who assisted in this study.

\section{CONFLICTS OF INTEREST}

All authors declare no conflicts of interest.

\section{FUND}

No fund was received for this work.

\section{REFERENCES}

ANRS collaborative study group on hepatocellular carcinoma (ANRS CO22 HEPATHER, CO12 CirVir and CO23 CUPILT cohorts) (2016). Lack of evidence of an effect of direct-acting antivirals on the recurrence of hepatocellular carcinoma: Data from three ANRS cohorts. Journal of Hepatology, 65(4): 734740 .
Baumert TF, Jühling F, Ono A, Hoshida Y (2017). Hepatitis C-related hepatocellular carcinoma in the era of new generation antivirals. BMC Medicine, 15(1): 52.

Bielen R, Moreno C, Van Vlierberghe $\mathrm{H}$, Bourgeois $\mathrm{S}$, Mulkay JP, Vanwolleghem T, Verlinden W, Brixco C, Decaestecker J, de Galocsy C, Janssens F, Van Overbeke L, Van Steenkiste C, D'Heygere F, Cool M, Wuyckens K, Nevens F, Robaeys G (2017). The risk of early occurrence and recurrence of hepatocellular carcinoma in hepatitis C-infected patients treated with direct-acting antivirals with and without pegylated interferon: A Belgian experience. Journal of Viral Hepatitis, 24(11): 976-981.

Chu PS, Nakamoto N, Taniki N, Ojiro K, Amiya T, Makita Y, Murata H, Yamaguchi A, Shiba S, Miyake R, Katayama T, Ugamura A, Ikura A, Takeda K, Ebinuma H, Saito H, Kanai T (2017). On-treatment decrease of NKG2D correlates to early emergence of clinically evident hepatocellular carcinoma after interferonfree therapy for chronic hepatitis C. PloS one, 12(6): e0179096.

Collins GS, Reitsma JB, Altman DG, Moons KG (2015). Transparent reporting of a multivariable prediction model for individual prognosis or diagnosis (TRIPOD): the TRIPOD statement. BMJ (Clinical Research ed.), 350: g7594.

Colombo M, Boccaccio V (2018). HCV therapy and risk of liver cancer recurrence: who to treat? Nature reviews. Gastroenterology \& Hepatology, 15(7): 392-393.

El Kassas M, Elbaz T, Salaheldin M, Abdelsalam L, Kaseb A, Esmat G (2019). Impact of treating chronic hepatitis $C$ infection with directacting antivirals on the risk of hepatocellular carcinoma: The debate continues - A minireview. Journal of Advanced Research, 17: 43-48.

El Kassas M, Funk AL, Salaheldin M, Shimakawa $Y$, Eltabbakh M, Jean K, El Tahan A, Sweedy AT, Afify S, Youssef NF, Esmat G, Fontanet A (2018). Increased recurrence rates of hepatocellular carcinoma after DAA therapy in a hepatitis C-infected Egyptian cohort: A comparative analysis. Journal of Viral Hepatitis, 25(6): 623-630.

European Association for the Study of the Liver (2018). EASL Clinical Practice Guidelines: Management of hepatocellular carcinoma. Journal of hepatology,69(1): 182-236.

European Association for the Study of the Liver (2017). EASL Recommendations on 
Treatment of Hepatitis C 2016. Journal of hepatology, 66(1): 153-194.

Guarino M, Viganò L, Ponziani FR, Giannini EG, Lai Q, Morisco F, Special Interest Group on Hepatocellular carcinoma and new anti-HCV therapies of the Italian Association for the Study of the Liver (2018). Recurrence of hepatocellular carcinoma after direct acting antiviral treatment for hepatitis $\mathrm{C}$ virus infection: Literature review and risk analysis. Digestive and Liver Disease: Official Journal of the Italian Society of Gastroenterology and the Italian Association for the Study of the Liver, 50(11): 1105-1114.

Kolly P, Waidmann O, Vermehren J, Moreno C, Vögeli I, Berg T, Semela D, Zeuzem S, Dufour JF (2017). Hepatocellular carcinoma recurrence after direct antiviral agent treatment: A European multicentre study. Journal of Hepatology, 67(4): 876-878.

Meissner EG, Wu D, Osinusi A, Bon D, Virtaneva K, Sturdevant D, Porcella S, Wang $H$, Herrmann $E$, McHutchison J, Suffredini AF, Polis $M$, Hewitt S, Prokunina-Olsson L, Masur H, Fauci AS, Kottilil S (2014). Endogenous intrahepatic IFNs and association with IFN-free HCV treatment outcome. The Journal of Clinical Investigation, 124(8): 3352-3363.

Minami T, Tateishi R, Nakagomi R, Fujiwara $N$, Sato M, Enooku K, Nakagawa H, Asaoka Y, Kondo Y, Shiina S, Koike K (2016). The impact of direct-acting antivirals on early tumor recurrence after radiofrequency ablation in hepatitis C-related hepatocellular carcinoma. Journal of Hepatology, 65(6): 1272-1273.

Ogawa E, Furusyo N, Nomura H, Dohmen K, Higashi N, Takahashi K, Kawano A, Azuma K, Satoh T, Nakamuta M, Koyanagi T, Kato M, Shimoda S, Kajiwara E, Hayashi J, Kyushu University Liver Disease Study (KULDS) Group (2018). Shortterm risk of hepatocellular carcinoma after hepatitis $C$ virus eradication following directacting anti-viral treatment. Alimentary Pharmacology \& Therapeutics, 47(1): 104113.

Piscaglia F, Granito A, Bolondi L (2018). DAAs for HCV and risk of hepatocellular carcinoma: current standpoint. The lancet. Gastroenterology \& Hepatology, 3(11): 736-738.

Reig M, Boix L, Bruix J (2017). The impact of direct antiviral agents on the development and recurrence of hepatocellular carcinoma. Liver International: Official Journal of the International Association for the Study of the Liver, 37 Suppl 1: 136-139.
Reig M, Mariño Z, Perelló $C$, Iñarrairaegui M, Ribeiro A, Lens S, Díaz A, Vilana R, Darnell A, Varela M, Sangro B, Calleja JL, Forns X, Bruix J (2016). Unexpected high rate of early tumor recurrence in patients with HCV-related HCC undergoing interferon-free therapy. Journal of Hepatology, 65(4): 719-726.

Sanduzzi-Zamparelli M, Boix L, Leal C, Reig M (2019). Hepatocellular Carcinoma Recurrence in HCV Patients Treated with Direct Antiviral Agents. Viruses, 11(5): 406.

Saraiya N, Yopp AC, Rich NE, Odewole M, Parikh ND, Singal AG (2018). Systematic review with meta-analysis: recurrence of hepatocellular carcinoma following direct-acting antiviral therapy. Alimentary Pharmacology \& Therapeutics, 48(2): 127-137.

Serti E, Chepa-Lotrea X, Kim YJ, Keane M, Fryzek N, Liang TJ, Ghany M, Rehermann B (2015). Successful Interferon-Free Therapy of Chronic Hepatitis C Virus Infection Normalizes Natural Killer Cell Function. Gastroenterology, 149(1): 190-200.

Sherman M (2008). Recurrence of hepatocellular carcinoma. The New England journal of medicine, 359(19): 2045-2047.

Tampaki M, Savvanis S, Koskinas J (2018). Impact of direct-acting antiviral agents on the development of hepatocellular carcinoma: evidence and pathophysiological issues. Annals of Gastroenterology, 31(6): 670-679.

Teng MW, Swann JB, Koebel CM, Schreiber RD, Smyth MJ (2008). Immune-mediated dormancy: an equilibrium with cancer. Journal of Leukocyte Biology, 84(4): 988-993.

van Wilgenburg B, Scherwitzl I, Hutchinson EC, Leng T, Kurioka A, Kulicke C, de Lara C, Cole S, Vasanawathana S, Limpitikul W, Malasit P, Young D, Denney L, STOP-HCV consortium, Moore, MD, Fabris P, Giordani MT, Oo YH, Laidlaw SM, Dustin LB, Klenerman P (2016). MAIT cells are activated during human viral infections. Nature communications, 7: 11653.

Villani R, Facciorusso A, Bellanti F, Tamborra R, Piscazzi A, Landriscina $M$, Vendemiale $G$, Serviddio G (2016). DAAs Rapidly Reduce Inflammation but Increase Serum VEGF Level: A Rationale for Tumor Risk during Anti-HCV Treatment. PloS One, 11(12): e0167934.

Virlogeux V, Pradat P, Hartig-Lavie K, Bailly F, Maynard M, Ouziel G, Poinsot D, Lebossé F, Ecochard M, Radenne S, Benmakhlouf S, Koffi J, Lack P, Scholtes C, Uhres AC, Ducerf C, Mabrut JY, Rode, A, Levrero M, Combet C, Zoulim F (2017). Direct-acting antiviral 
therapy decreases hepatocellular carcinoma recurrence rate in cirrhotic patients with chronic hepatitis C. Liver International: Official Journal of the International Association for the Study of the Liver, 37(8): 1122-1127.
Waring JF, Dumas EO, Abel S, Coakley E, Cohen DE, Davis JW, Podsadecki T, Dutta S (2016). Serum miR-122 may serve as a biomarker for response to direct acting antivirals: effect of paritaprevir/R with dasabuvir or ombitasvir on miR-122 in HCV-infected subjects. Journal of Viral Hepatitis, 23(2): 96-104. 